\title{
Dielectric elastomer generators that stack up
}

\author{
T G McKay ${ }^{1,2}$, S Rosset ${ }^{1}$, I A Anderson ${ }^{2}$ and H Shea ${ }^{1}$ \\ ${ }^{1}$ LMTS, École polytechnique fédérale de Lausanne (EPFL), Switzerland \\ ${ }^{2}$ The Biomimetics Lab, Auckland Bioengineering Institute, The University of Auckland, New Zealand \\ E-mail: tomgmckay@gmail.com
}

Received 7 August 2014, revised 23 October 2014

Accepted for publication 10 November 2014

Published 8 December 2014

\begin{abstract}
This paper reports the design, fabrication, and testing of a soft dielectric elastomer power generator with a volume of less than $1 \mathrm{~cm}^{3}$. The generator is well suited to harvest energy from ambient and from human body motion as it can harvest from low frequency (sub-Hz) motions, and is compact and lightweight. Dielectric elastomers are highly stretchable variable capacitors. Electrical energy is produced when the deformation of a stretched, charged dielectric elastomer is relaxed; like-charges are compressed together and opposite-charges are pushed apart, resulting in an increased voltage. This technology provides an opportunity to produce soft, high energy density generators with unparalleled robustness. Two major issues block this goal: current configurations require rigid frames that maintain the dielectric elastomer in a prestretched state, and high energy densities have come at the expense of short lifetime. This paper presents a selfsupporting stacked generator configuration which does not require rigid frames. The generator consists of 48 generator films stacked on top of each other, resulting in a structure that fits within an $11 \mathrm{~mm}$ diameter footprint while containing enough active material to produce useful power. To ensure sustainable power production, we also present a mathematical model for designing the electronic control of the generator which optimizes energy production while limiting the electrical stress on the generator below failure limits. When cyclically compressed at $1.6 \mathrm{~Hz}$, our generator produced $1.8 \mathrm{~mW}$ of power, which is sufficient for many low-power wireless sensor nodes. This performance compares favorably with similarly scaled electromagnetic,

piezoelectric, and electrostatic generators. The generator's small form factor and ability to harvest useful energy from low frequency motions such as tree swaying or shoe impact provides an opportunity to deliver power to remote wireless sensor nodes or to distributed points in the human body without the need for costly periodic battery replacement.
\end{abstract}

Keywords: energy harvester, dielectric elastomer, electroactive polymer, DEG, EAP, small scale, internet of things

(Some figures may appear in colour only in the online journal)

\section{Introduction}

The internet of things is revolutionizing the way we interact with objects, and the way in which objects themselves interact [1]. One exciting subset of the internet of things is wireless sensor networks. With applications such as sensors monitoring the health of a building or bridge, the activity and performance of an athlete, or transducers to monitor wildlife populations, wireless sensor networks provide an opportunity to obtain an enhanced awareness of both our local and remote environments. One major advantage of a wireless sensor network is that the cost and inconvenience of wiring between nodes is eliminated $[2,3]$.

The advantages of wireless sensor networks can be offset if frequent battery replacement or charging is required. One solution is to integrate a compact energy harvester into the wireless sensor nodes. Ongoing improvements in sensor and wireless communication technologies have included substantial reductions in power consumption. Thanks to this, a device that can generate $1 \mathrm{~mW}$ and fit within $1 \mathrm{~cm}^{3}$ envelope would allow the powering of a very broad range of devices.

Dielectric elastomer generators (DEGs), a class of electroactive polymers, are highly deformable variable capacitors 

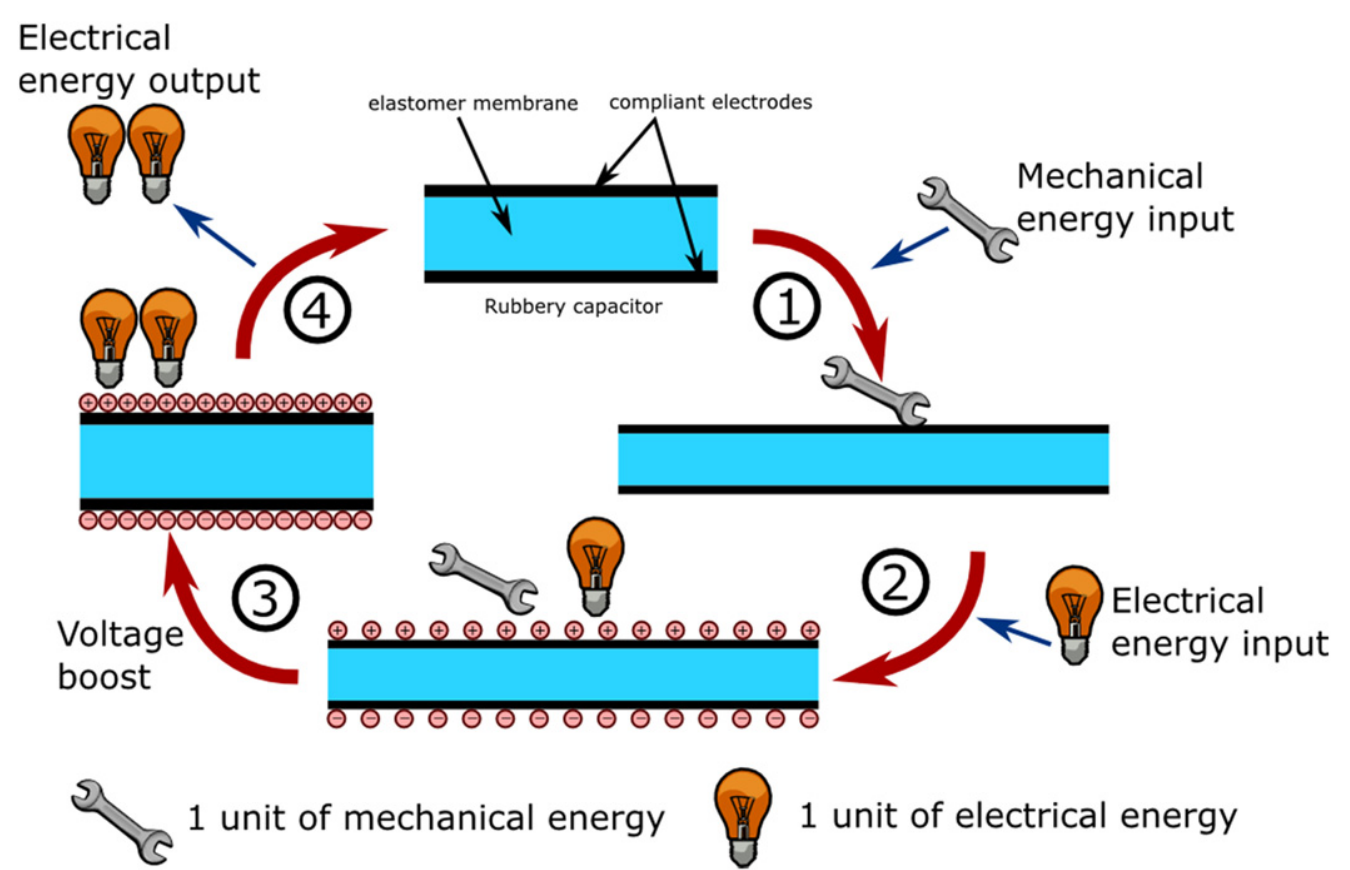

\section{1 unit of electrical energy}

Figure 1. The DEG energy harvesting cycle. (1) Mechanical energy is used to stretch the generator; (2) the generator is electrically charged; (3) the generator is mechanically relaxed which pushes opposite charges apart and compresses like charges together, this action boosts the electrical energy of the charges; (4) the charges are then extracted in this higher energy state.

that show great promise for sub- $\mathrm{cm}^{3}$ scale energy harvesters. Electrical energy is produced when the deformation of a stretched, charged DEG is relaxed; like-charges are compressed together and opposite-charges are pushed apart, resulting in an increased voltage (see figure 1). DEG lend themselves well to low complexity, small-scale energy harvesters because the energy harvesting mechanism is theoretically scale invariant, they can be directly coupled to rectilinear motions [4], they can produce high energy densities [5, 6], and can harvest energy efficiently from a wide range of frequencies without the requirement for operation at or near a resonant frequency. Much energy harvesting research has focused on resonant spring-mass systems, optimized for vibrations often at a predetermined frequency [7]. A key benefit of DEG is that they can harvest from extremely low frequency motion (e.g. the swaying of a tree branch [8] and ocean waves [9-11]) and by virtue of their softness can obtain energy from every-day human motions unobtrusively $[12,13]$.

Although complete DEG systems, including the electronics required for controlling the energy harvesting cycle, have been fabricated from soft rubbers [14], they have been based on prestretched films with thicknesses of tens to hundreds of micrometers [4-6]. These thin film generators require rigid frames to maintain their desired shape and high level of prestretch. The framing is an additional part that is rigid and relatively heavy, so it reduces the generator's energy density, deformability, simplicity, and introduces potentially catastrophic stress concentrations.

In this paper we present a stacked membrane generator configuration which allows the fabrication of self-supporting generators that do not require rigid frames. The stacked configuration also allows a large quantity of generator material to be fabricated onto a much smaller footprint than that achievable using a single thin membrane. Another advantage of the stack generator configuration is that, when compressed, the generator membranes take on a state that is equivalent to equi-biaxial tension. Equi-biaxial tension has been shown to be an ideal deformation mode for DEG because, for a given area stretch, it maximizes the capacitance change [6].

This paper describes the manufacturing process used to fabricate the stack generator; a model of the energy harvesting cycle is then presented which determines operating conditions to maximize energy output using our charge control strategy. Experimental results, which use the optimized control strategy, are then presented.

\section{Methods}

Our generator consisted of $48,65 \mu \mathrm{m}$ thick, $11 \mathrm{~mm}$ diameter DEG layers stacked on top of each other and electrically connected in parallel. The generator stack was then sandwiched between $3 \mathrm{~mm}$ thick silicone end caps, forming a structure with a height of $9 \mathrm{~mm}$ and a volume of $0.86 \mathrm{~cm}^{3}$ (see figure 2). The end caps were included because the most convenient mechanism for coupling a stack to a source of mechanical deformation is to adhere the ends of the stack between two structures. The constrained ends do not deform and the structure barrels when compressed. Thus the end caps ensure that the active DEG zone is essentially homogeneously deformed [3, 15], which means that the electric field is approximately the same for each layer of the stack generator. It is advantageous for the electric field to be uniform because 


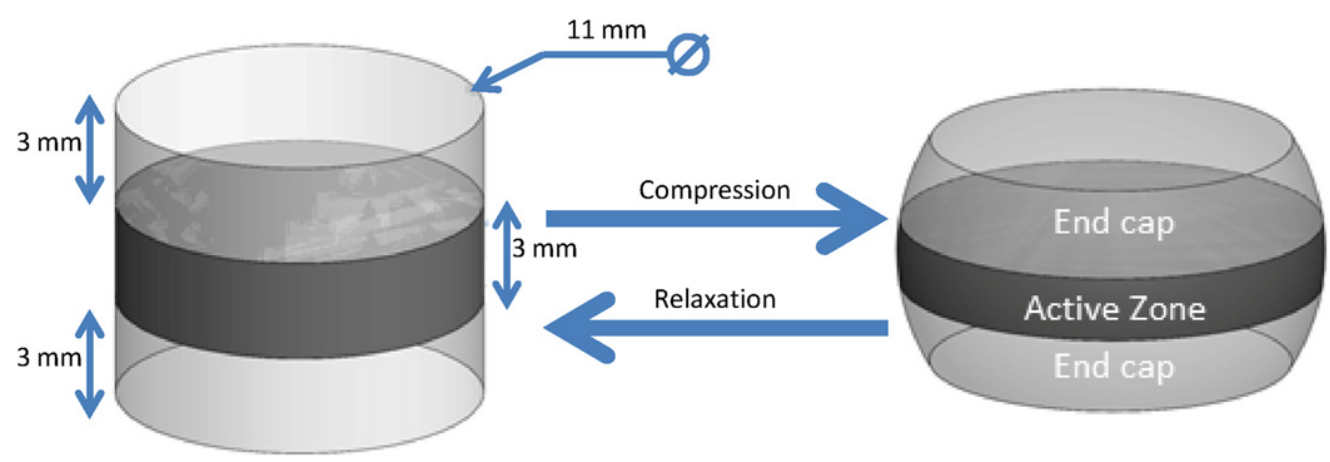

Figure 2. The stack DEG is coupled to a mechanical energy source by adhering its ends between two bodies that move relative to each other. The active generator zone is deformed when the stack is compressed. The end caps do not deform as much as the the active zone because of the fixed boundary condition between the generator stack and the mechanical energy source.

\section{Pad-print electrodes onto silicone film}

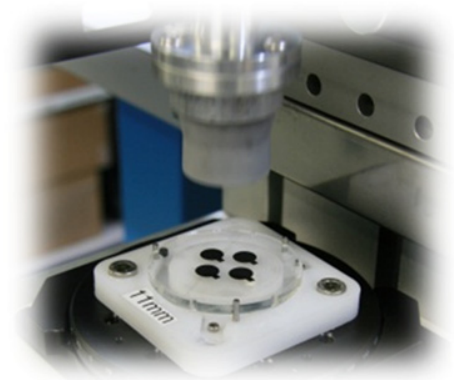

\section{Laser-cut the perimeter of the stacks}

\section{Plasma-bond layers together}

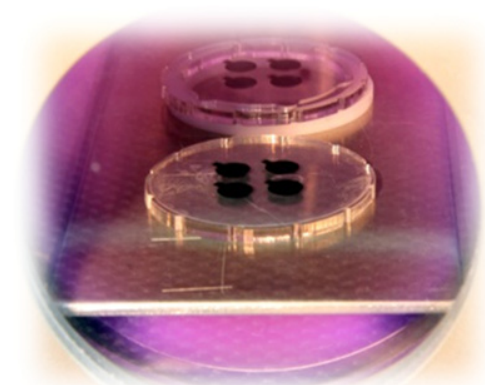

4. Adhere sub-stacks together, attach end caps, and apply interconnecting electrodes
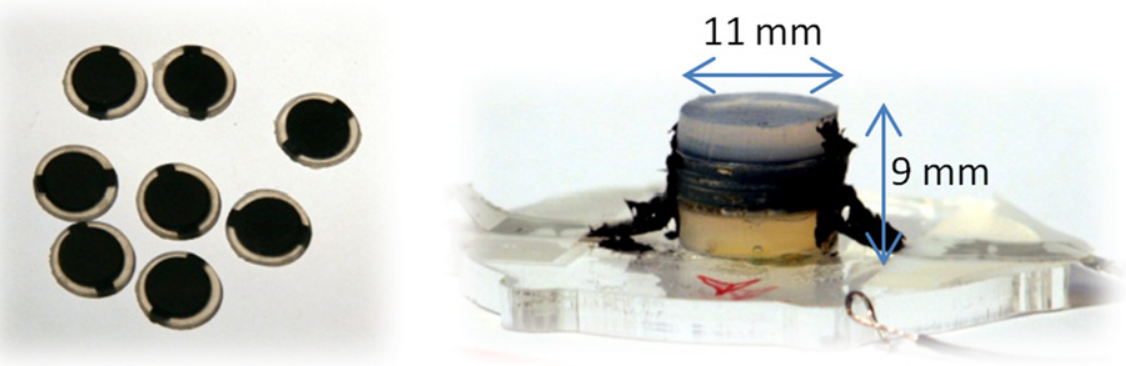

Figure 3. An overview of the process which was developed for fabrication of stacked DEG. (1) Four carbon-based electrodes were padprinted onto the silicone dielectric layers; (2) a plasma bonder was used to adhere 12 electroded dielectric layers together; (3) each of the four sub-stacks were cut free using a laser-cutter; (4) the sub-stacks were glued on top of each other using a silicone glue and a carbon electrode mix electrically connected each sub-stack in parallel.

DEG energy production increases with electric field. If the electric field is not uniform, then regions of the generator operating at lower electric fields will contribute to a reduced energy density.

An overview of our fabrication process is given in figure 3. $65 \mu \mathrm{m}$ thick Wacker Chemie AG Elastosil® silicone membranes were used as the dielectric. Electrodes consisting of $90 \%$ by weight Silbione LSR 4305 silicone, Ketjenblack EC-300J carbon black (Akzo-Nobel) (5\% by weight), and Cabot Black Pearl 2000 carbon black (5\% by weight) were applied to the membranes using a TPM-101 pad printer from Teca-Print. A Diener Zepto plasma system was then used to bond the layers together with a short exposure to oxygen plasma, until four sub-stacks of 12 active membranes were formed. Once the sub-stacks were formed, a Trotec Speedy
300 laser cutter was used to cut the sub-stacks to shape. The substacks were then bonded together to form one large generator before LSR-20 silicone (factor 2) endcaps were bonded to the stack. The layers were then interconnected using the silicone/Ketjenblack/Cabot carbon black electrode mix.

The stack generator was tested using the energy harvesting circuit illustrated in figure 4 , which controls the charge on the generator using an energy harvesting cycle that has been previously studied both theoretically and experimentally $[5,6,12,16]$. The generator transfers charge from a priming source up to a higher voltage where the energy was supplied to a load. Thus our energy harvester provides a gain in electrical energy by boosting the voltage of the charge. We replicated the approach of Huang et al who used a zener diode as the load (simulating an infinitely large harvesting 


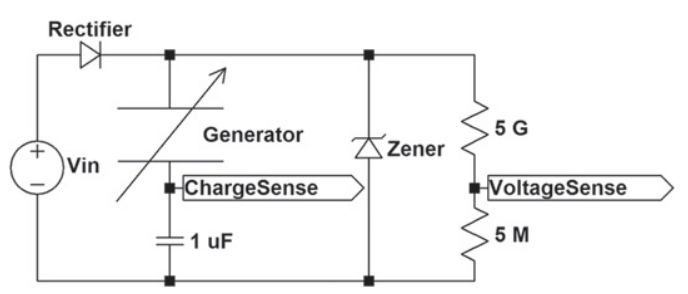

Figure 4. A schematic of the circuit used for analysis of the stack generator. The generator is placed in series with a capacitor $(1 \mu \mathrm{F})$ whose voltage (ChargeSense) tracks the charge state of the generator. The generator was charged using a high voltage power supply $\left(V_{\text {in }}\right)$ through a rectifier. The zener diode fixed the maximum voltage across the generator. The voltage across the generator was measured using a $5 \mathrm{G}: 5 \mathrm{M}$ voltage divider (VoltageSense).

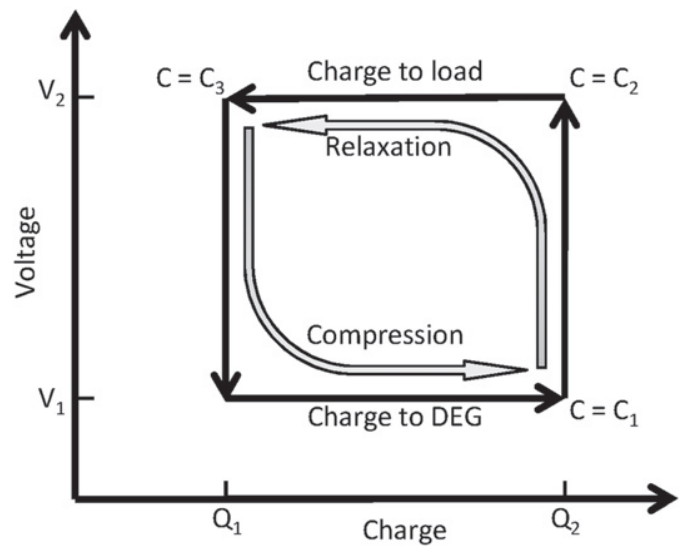

Figure 5. A schematic of the priming circuit's energy harvesting cycle mapped on the voltage charge plane. The solid arrows follow the voltage/charge state of the DEG throughout the cycle, whereas the curved arrows demonstrate the mechanical state. $C_{1}$ refers to the capacitance of the generator when it is fully compressed. The capacitance of the generator reduces as it is decompressed and when it's capacitance is reduced to $C_{2}$, it's voltage reaches the breakdown voltage of the zener array $\left(V_{2}\right)$. As the generator continues to decompress to a capacitance of $C_{3}$, charge is transferred to the zener array, which is our simulated load.

capacitor) [6]. When the voltage reaches the breakdown voltage of the zener array, charge is transferred from the generator to the zener load which consisted of several $1 \mathrm{~N} 5388 \mathrm{BG} 200 \mathrm{~V}$ zener diodes in series. The generator was primed to $V_{\text {in }}$ using a Biomimetics Lab (Auckland) EAP controller power supply. The voltage across the DEG was measured using a $5 \mathrm{G} \Omega: 5 \mathrm{M} \Omega$ resistor ladder. The charge on the generator was measured using a $1 \mu \mathrm{F}$ current integration capacitor.

With reference to our circuit in figure 4, the generator's input voltage $V_{\text {in }}$ and the breakdown voltage of the zener array need to be carefully chosen because they dictate the charge state of the DEG. The energy harvesting cycle produced using the circuit is schematically described on the voltage charge plane in figure 5 . The greater the area of the energy harvesting cycle, the more energy is harvested $[5,16,17]$. We were interested in optimizing the amount of energy that we can harvest from a specific generator.
A model was developed to determine the operating voltages, $V_{1}$ and $V_{2}$, that optimize energy production without driving the generator to failure.

The energy production in a single cycle is the area of the energy harvesting cycle on the voltage charge plane in figure 5, as described in equation (1) [6]

$$
W_{\text {elect }}=\left(Q_{2}-Q_{1}\right)\left(V_{2}-V_{1}\right) \text {. }
$$

As the capacitance decreases from $C_{1}$ to $C_{2}$, the charge remains constant at $Q_{2}$ and we can therefore relate the input voltage $V_{1}$ to the zener breakdown voltage $V_{2}$ using equation (2):

$$
\begin{aligned}
Q & =C V, \\
\therefore C_{1} V_{1} & =C_{2} V_{2}, \\
\therefore V_{1} & =\frac{C_{2} V_{2}}{C_{1}} .
\end{aligned}
$$

When the DEG capacitance decreases from $C_{2}$ to $C_{3}$ the voltage is held constant at $V_{2}$ so we can relate the charge on the fully relaxed generator $Q_{1}$ to the stretched generator's charge $Q_{2}$ using equation (3):

$$
\begin{aligned}
V & =\frac{Q}{C}, \\
\therefore \frac{Q_{2}}{C_{2}} & =\frac{Q_{1}}{C_{3}} .
\end{aligned}
$$

We can then get an expression for the energy generated in one compression cycle of a generator that has a stretched and relaxed capacitance of $C_{1}$ and $C_{3}$, respectively, by substituting equations (2) and (3) into (1):

$$
W_{\text {elect }}=Q_{2} V_{2}\left(1-\frac{C_{3}}{C_{2}}\right)\left(1-\frac{C_{2}}{C_{1}}\right) .
$$

The electric field increases when $Q$ is held constant and the capacitance is reduced because the voltage climbs at a greater rate than the thickness of the generator increases [18]. The electric field drops when the voltage is held constant and the capacitance is reduced, thus the highest electric field occurs when $C=C_{2}$. At $C_{2}$ the voltage is equal to $V_{2}$ and the thickness, $t_{2}$, can be found by relating $C_{3}$ to $C_{2}$ as follows:

$$
t_{2}=\sqrt{\frac{C_{3}}{C_{2}}} t_{0} .
$$

And we can find the generator's maximum electric field by calculating the field when the generator is at a capacitance of $\mathrm{C}_{2}$ :

$$
E_{\max }=\frac{V_{2}}{t_{2}} .
$$

Equations (7) and (8) then give us $V_{2}$ and $Q_{2}$ required to operate at $E_{\text {max }}$ :

$$
V_{2}=E_{\max } t_{2}=E_{\max } \sqrt{\frac{C_{3}}{C_{2}}} t_{0},
$$



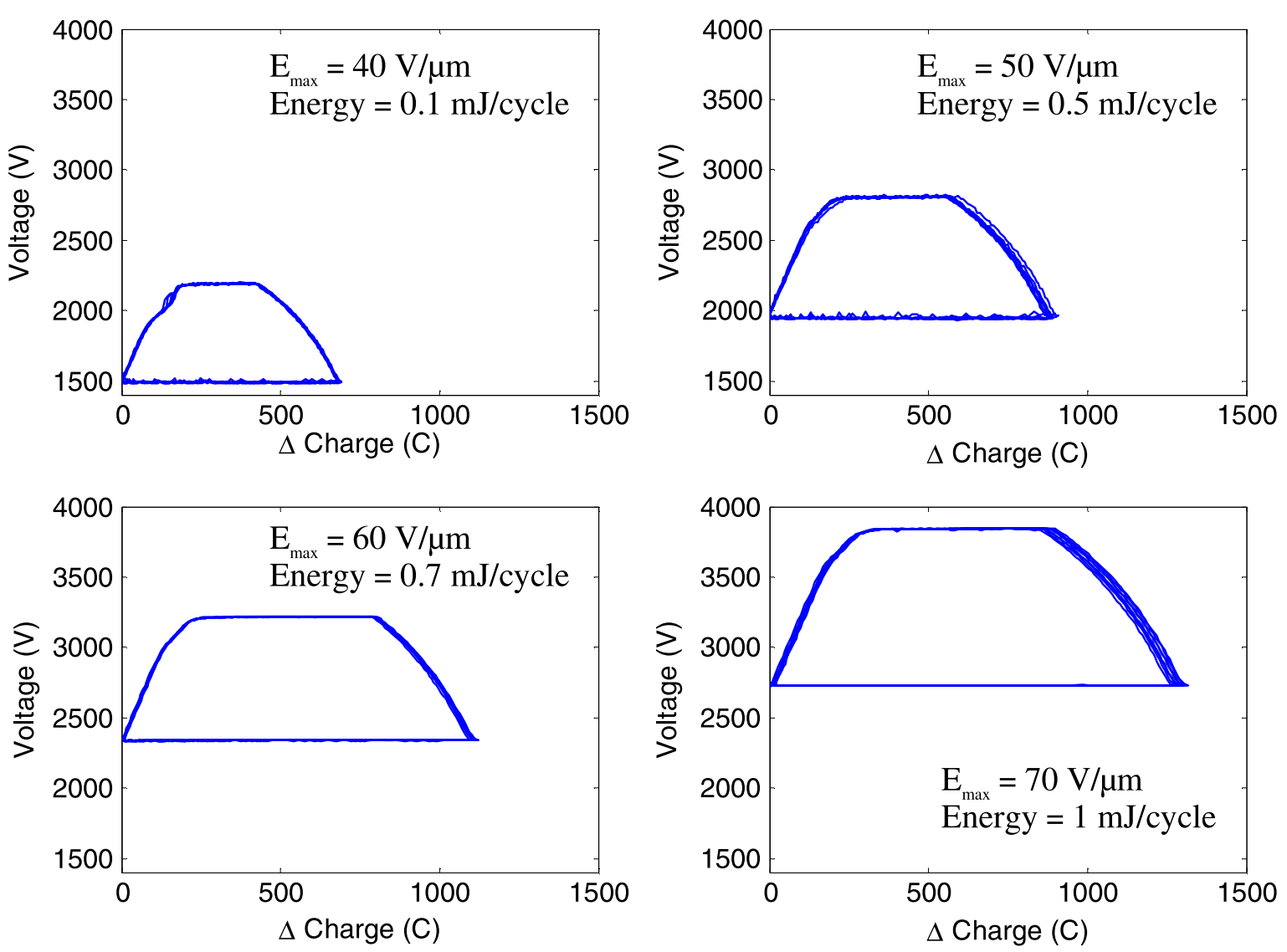

Figure 6. Voltage versus change in charge measured during nine compression cycles of our generator at a maximum electric field of

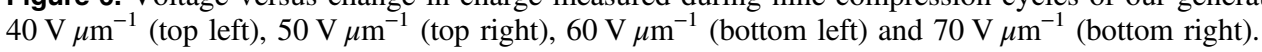

$$
Q_{2}=C_{2} V_{2}=C_{2} E_{\max } \sqrt{\frac{C_{3}}{C_{2}}} t_{0} .
$$

Substituting (7) and (8) into (4) we get an equation that describes the energy harvesting cycle that operates at a specified maximum electric field of $E_{\text {max }}$ :

$$
W_{\text {elect }}=C_{3} E_{\max }^{2} t_{0}^{2}\left(1-\frac{C_{3}}{C_{2}}\right)\left(1-\frac{C_{2}}{C_{1}}\right) .
$$

To find our optimal operating conditions we want to find $C_{2}$ that maximizes electrical energy production. To find this maximum, we need to differentiate equation (9) with respect to $C_{2}$ and set the derivative to zero:

$$
\begin{aligned}
\frac{\mathrm{d} W_{\text {elect }}}{\mathrm{d} C_{2}} & =\frac{E_{\mathrm{max}}^{2} t_{0}^{2} C_{3}^{2}}{C_{2}^{2}}-\frac{E_{\mathrm{max}}^{2} t_{0}^{2} C_{3}}{C_{1}}=0, \\
\therefore C_{2} & =\sqrt{C_{3} C_{1}} .
\end{aligned}
$$

Given that we know the thickness of the un-deformed generator $\left(t_{0}\right)$, the maximum and minimum generator capacitance ( $C_{1}$ and $C_{3}$, respectively), and the maximum desired operating field $\left(E_{\max }\right)$ we can use equations (10), (7), and (2) to determine the optimal values for operating voltages $V_{1}$ and $V_{2}$ for the energy harvesting cycle used in this study.

The stacked generator was tested by cyclically compressing the stack by $2.75 \mathrm{~mm}$ at a frequency of $0.47 \mathrm{~Hz}$ using an Instron 3342 dynamic test rig. This deformation was enough to approximately double the capacitance from a fully compressed value $\left(C_{1}\right)$ of $1.15 \mathrm{nF}$ to a relaxed value $\left(C_{3}\right)$ of $0.56 \mathrm{nF}$. The tests were performed at maximum electric fields of $40 \mathrm{~V} \mu \mathrm{m}^{-1}, 50 \mathrm{~V} \mu \mathrm{m}^{-1}, 60 \mathrm{~V} \mu \mathrm{m}^{-1}$, and $70 \mathrm{~V} \mu \mathrm{m}^{-1}$ which, as calculated using our model, corresponded to the following input voltages $\left(V_{1}\right)$ and zener breakdown voltages $\left(V_{2}\right)$.

\section{Results and discussion}

The measured voltage versus charge relationships are given in figure 6 for nine cycles operating at each of the maximum electric fields listed in table 1. A notable feature of these plots is that the energy harvesting cycle does not follow the expected square perimeter as shown in the schematic of figure 5. The sloped sides of the energy harvesting cycle indicate that a significant amount of charge is being drawn off the generator during the stages of the energy harvesting cycle when the DEG charge is intended to be fixed. This charge reduction is due to the current drawn by the $5 \mathrm{G} \Omega$ sensor which as illustrated in figure 4, that is placed in parallel with the DEG. Leakage current through the zener array will also contribute to charge losses from the generator.

The charge loss mechanisms that we have identified are voltage dependent and the losses do not depend on the rate at which the DEG is deformed. Thus, with a goal of enhancing energy production per stroke, the generator was cycled at a 
Table 1. Operating conditions used to operate the DEG at maximum electric fields ranging from $40 \mathrm{~V} \mu \mathrm{m}^{-1}$ to $70 \mathrm{~V} \mu \mathrm{m}^{-1}$.

\begin{tabular}{lll}
\hline $\begin{array}{l}\text { Maximum elec- } \\
\text { tric field }\end{array}$ & $\begin{array}{l}V_{1} \text { (charging } \\
\text { voltage) }\end{array}$ & $\begin{array}{l}V_{2} \text { (discharging } \\
\text { voltage) }\end{array}$ \\
\hline $40 \mathrm{~V} \mu \mathrm{m}^{-1}$ & $1.5 \mathrm{kV}$ & $2.2 \mathrm{kV}$ \\
$50 \mathrm{~V} \mu \mathrm{m}^{-1}$ & $1.95 \mathrm{kV}$ & $2.8 \mathrm{kV}$ \\
$60 \mathrm{~V} \mu \mathrm{m}^{-1}$ & $2.3 \mathrm{kV}$ & $3.2 \mathrm{kV}$ \\
$70 \mathrm{~V} \mu \mathrm{m}^{-1}$ & $2.7 \mathrm{kV}$ & $3.8 \mathrm{kV}$ \\
\hline
\end{tabular}

faster rate of $0.9 \mathrm{~Hz}$ and $1.6 \mathrm{~Hz}$. As shown in figure 7, the sides of the voltage charge plot are steeper when operating at the higher frequencies, illustrating that the charge losses are less significant.

As described by Huang et al the energy produced by the generator in a single cycle was found by computing the area within the voltage-charge envelope [6]. The average energy produced in a single cycle for each operating electric field and investigated frequency is provided in figure 8 . Consistent with previous studies $[4,19,20]$ and equation (9), energy production increased with the operating electric field.

The generator produced $1.1 \mathrm{~mJ}$ in a single cycle when operating at an electric field of $70 \mathrm{~V} \mu \mathrm{m}^{-1}$. When the Instron cyclically deformed the DE generator at $1.6 \mathrm{~Hz}$, the $1.1 \mathrm{~mJ}$ per cycle corresponded to an average power production of $1.8 \mathrm{~mW}$; considering our generator has a volume of $0.86 \mathrm{~cm}^{3}$ (including end-caps), we achieved a power density of $2.1 \mathrm{~mW} \mathrm{~cm}^{-3}$.

The performance of our energy harvester compares well with other technologies. For instance, Hudak and Amatucci provide a review of small scale energy harvesters, and none of the sub- $\mathrm{cm}^{3}$ electromagnetic, electrostatic, or piezoelectric energy harvesters produced over $1.8 \mathrm{~mW}$ with a stimulus frequency of less than $50 \mathrm{~Hz}$ [21]. In fact they summarized the power density of 19 small-scale energy harvesters, and only three research groups reported on small-scale energy harvesters with a power density of more than $2.1 \mathrm{~mW} \mathrm{~cm}^{-3}$. All three energy harvesters that exceeded the power density of our device were mechanically stimulated at frequencies greater than $322 \mathrm{~Hz}$ [21], thus occupying a different design space from our low frequency energy harvester.

Figure 8 highlights that our generator performance was similar to that predicted by our model, with the predicted energy production generally slightly out-performing the measured generator performance. Such a discrepancy between the model and the fabricated device are expected as the model assumes lossless operation. However, the model qualitatively tracks the trend of our data and is therefore a sufficient representation of the real system to use as a tool for assisting the design of the generator's operating conditions.

We optimized the operating conditions of our stacked generator, so a comparison between the performance of our generator and the best reported performance is appropriate. A common parameter for comparing the performance of energy harvesters is the energy density, which is the amount of energy that is produced per unit mass of the energy harvester in one stroke. Our energy harvester, with a mass of $0.32 \mathrm{~g}$ (excluding the mass of the end-caps and priming electronics) and peak energy output of $1.1 \mathrm{~mJ}$ achieved an energy density of $3.5 \mathrm{~mJ} \mathrm{~g}^{-1}$ stroke.

This performance is low compared to the greatest energy density recorded for DE-generators of $560 \mathrm{~mJ} \mathrm{~g}^{-1}$ stroke reported by Huang et al [6]. However Huang et al who were set on breaking the world record for energy density without regard for reliable operation, achieved their high energy density using a different material (3M VHB4905) which they

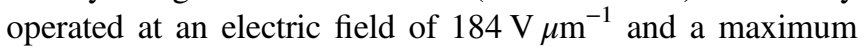
capacitance of $76 \mathrm{nF}$ and minimum capacitance of $0.19 \mathrm{nF}$. Under these conditions, their generator lasted less than ten cycles.

Our focus has been on the development of a compact device that could be used as a power source to supplement or replace batteries. To be a genuine alternative to batteries, the generator must be reliable. To achieve sufficient reliability, our generator was operated well within its failure limits. For instance, in preliminary tests, we were able to drive dielectric elastomers fabricated from the same Wacker Elastosil material up to electric fields of $90 \mathrm{MV} \mathrm{m}^{-1}$ before they would break down. Limiting our operating field below $70 \mathrm{MV} \mathrm{m}^{-1}$ and selecting mechanical operating conditions that restricted the material's linear strain below $20 \%$ allowed us to produce all of the results presented in this paper from a single generator. Further steps can be taken to improve reliability such as the inclusion of properly designed end caps that will promote more homogeneous deformation across the active layers of a DEG [22] thus promoting greater efficacy for energy harvesting by enabling the DEG to work at higher fields. Furthermore, careful end-cap design can improve the generator's energy density. For instance we fabricated a second generator that had the same dimensions as the one described in this study, except it consisted of 128 layers instead of 48 (thus the end-caps contributed a smaller portion of the generator's total height). The 128 layer generator was compressed, doubling its capacitance at $0.9 \mathrm{~Hz}$ to produce $5.4 \mathrm{~mW}$. This corresponded to a power density of $3.8 \mathrm{~mW} \mathrm{~cm}^{-3}$, which is an $81 \%$ improvement on our 48 layer generator. This illustrates that substantial improvements to generator performance are possible within a suitable operating envelope.

In summary, our work illustrates that one can reliably extract power at a scale that is useful for applications such as wireless sensor networks. Careful attention to design such as the provision for end caps and the stack's compression mode of operation will contribute to improved reliability. Good reliability will enhance confidence that we believe will lead to a widespread uptake of this technology for portable energy harvesting solutions in a power hungry world.

\section{Conclusions}

We have fabricated and characterized a miniature soft dielectric elastomer energy harvester which generated $1.8 \mathrm{~mW}$ when excited at a frequency of $1.6 \mathrm{~Hz}$. The operating voltages were optimized based on an analytical model of the 

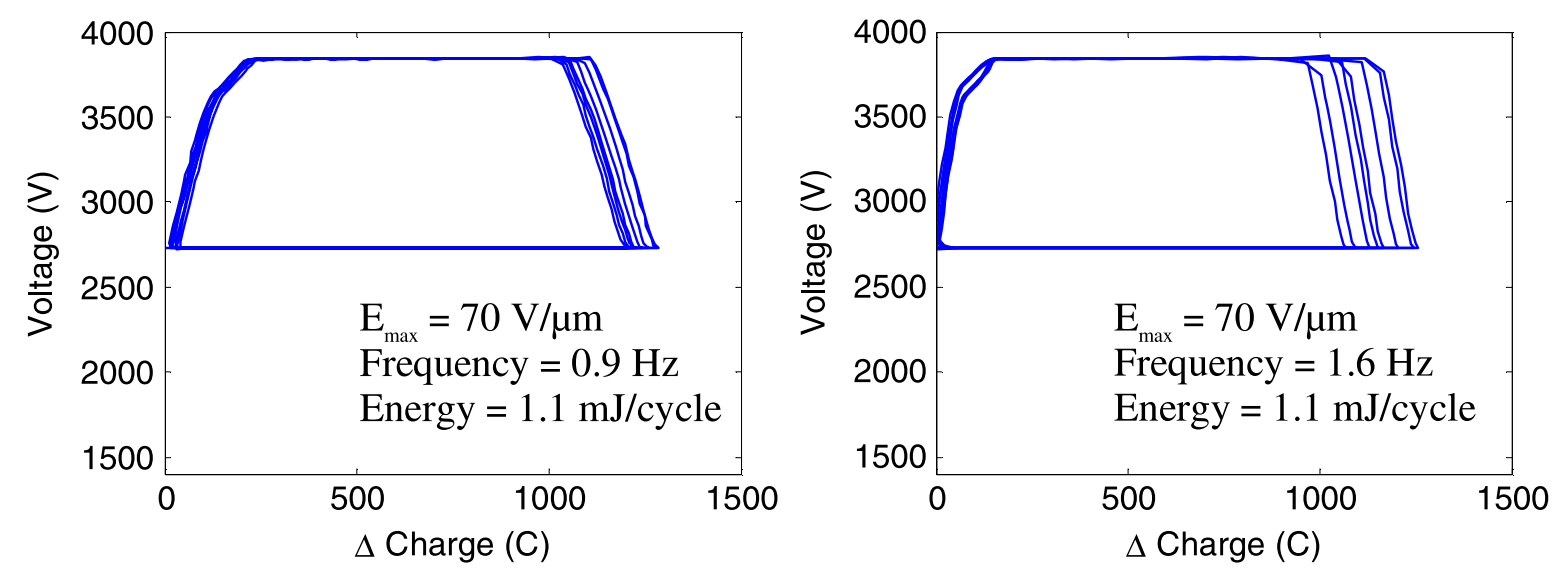

Figure 7. Voltage versus change in charge measurements during nine compression cycles of our generator at a maximum electric field of $70 \mathrm{~V} \mathrm{~m}^{-1}$ and cycling frequency of $0.9 \mathrm{~Hz}$ (left) and $1.6 \mathrm{~Hz}$ (right).

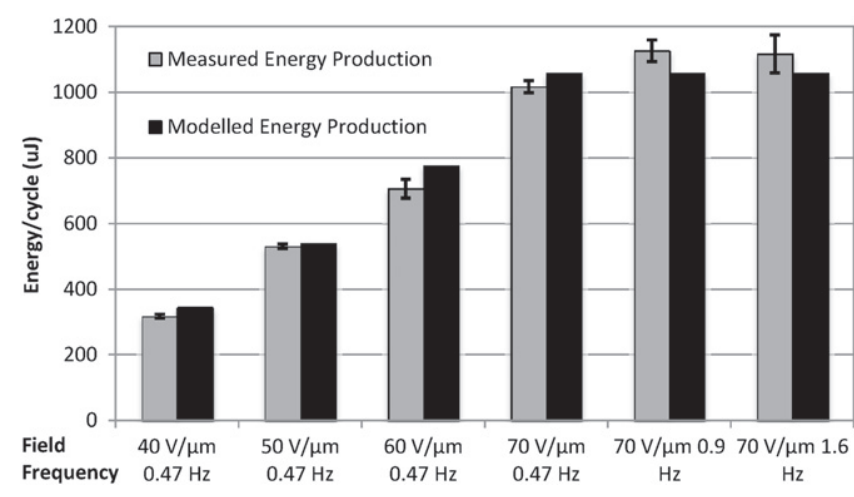

Figure 8. Energy produced by the stack DEG at electric fields ranging from $40 \mathrm{~V} \mu \mathrm{m}^{-1}$ to $70 \mathrm{~V} \mu \mathrm{m}^{-1}$ when operated at $0.47 \mathrm{~Hz}$ and when operated at an electric field of $70 \mathrm{~V} \mu \mathrm{m}^{-1}$ and frequency of $0.9 \mathrm{~Hz}$ and $1.6 \mathrm{~Hz}$. The error bars represent \pm 1 standard deviation.

charging cycle. The $1.8 \mathrm{~mW}$ produced by our miniature generator is at a useful level for a broad range of applications, including activity trackers, athletics, and wireless sensor networks. One milliwatt is sufficient power for many types of wireless sensor nodes. Ten years ago already, Rhee et al developed wireless sensor network nodes that consumed $300 \mu \mathrm{W}$ to transmit 1 packet of sensor data per second [3]. Furthermore the power output of electroactive polymer energy harvesters scales linearly with frequency, so power hungry nodes could be powered using a stacked DEG like the one described here if a higher frequency energy source was available.

The combination of our generator's small form factor, simple coupling mechanism, and ability to harvest useful energy from low frequency motions such as arm or leg swinging or tree swaying provides an opportunity to deploy complex unobtrusive wireless sensor nodes without the need for periodic, costly battery replacement. We previously used DEGs to harvest energy from a tree branch swaying in the breeze [8] for example to power wireless sensor nodes that provide an alarm if a forest fire develops. We have also embedded the stack configuration presented in this paper into the sole of a shoe (see URL: http://www.youtube.com/watch? $\mathrm{v}=\mathrm{G} 1 \mathrm{MAv}$-_lqg) to harvest energy dissipated during heel- strike. The higher energy density, long lifetime, and compliance of the miniature stacked generator we report here enables energy harvesting to be used widely in wearable and environmental monitoring applications.

\section{Acknowledgments}

This work was partially funded by the Swiss National Science Foundation grant \# 200020_140394 and the New Zealand Royal Society's Marsden Fund grant \# UOA1211. The authors thank Dr Andreas Köllnberger from Wacker Chemie AG for giving us sheets of Elastosil ${ }^{\circledR}$ silicone membrane, which were used to make the generators.

\section{References}

[1] Miorandi D, Sicari S, De Pellegrini F and Chlamtac I 2012 Internet of things: vision, applications and research challenges Ad Hoc Netw. 10 1497-516

[2] Yunxia C and Qing Z 2005 On the lifetime of wireless sensor networks IEEE Commun. Lett. 9 976-8

[3] McKay T G, Rosset S, Anderson I A and Shea H 2013 An electroactive polymer energy harvester for wireless sensor networks J. Phys.: Conf. Ser. 476012117

[4] McKay T G, O'Brien B, Calius E and Anderson I 2010 Selfpriming dielectric elastomer generators Smart Mater. Struct. 19055025

[5] Kaltseis R, Keplinger C, Baumgartner R, Kaltenbrunner M, Li T, Machler P, Schwdiauer R, Suo Z and Bauer S 2011 Method for measuring energy generation and efficiency of dielectric elastomer generators Appl. Phys. Lett. 99162904

[6] Huang J, Shian S, Suo Z and Clarke D R 2013 Maximizing the energy density of dielectric elastomer generators using equibiaxial loading Adv. Funct. Mater. 23 5056-61

[7] Beeby S P, Tudor M J and White N M 2006 Energy harvesting vibration sources for microsystems applications Meas. Sci. Technol. 17 R175

[8] Anderson I A, Ieropoulos I A, McKay T, O'Brien B and Melhuish C 2011 Power for robotic artificial muscles IEEE Trans. Mech. 16 107-11

[9] Jean P, Wattez A, Ardoise G, Melis C, Van Kessel R, Fourmon A, Barrabino E, Heemskerk J and Queau J P 2012 
Standing wave tube electro active polymer wave energy converter Electroactive Polym. Actuators Devices (EAPAD) 2012 83400C-21C

[10] Prahlad H, Kornbluh R, Pelrine R, Stanford S, Eckerle J and Oh S 2005 Polymer power: dielectric elastomers and their applications in distributed actuation and power generation Proc. ISSS (Bangalore, India) p 100

[11] Waki M, Chiba S, Kornbluh R, Pelrine R and Kunihiko U 2008 Electric power from artificial muscles OCEANS 2008-MTS/ IEEE Kobe Techno-Ocean pp 1-3

[12] Jean-Mistral C and Basrour S 2010 Scavenging energy from human motion with tubular dielectric polymer Electroactive Polym. Actuators Devices (EAPAD) 2010 764209-12

[13] Pelrine R, Kornbluh R D, Eckerle J, Jeuck P, Oh S, Pei Q and Stanford S 2001 Dielectric elastomers: generator mode fundamentals and applications Proc. SPIE pp 148-56

[14] McKay T G, O'Brien B M, Calius E P and Anderson I A 2011 Soft generators using dielectric elastomers Appl. Phys. Lett. 98142903

[15] Foerster F and Schlaak H F 2014 Optimized deformation behavior of a dielectric elastomer generator Proc. SPIE 9056 905637
[16] Koh S J A, Zhao X and Suo Z 2009 Maximal energy that can be converted by a dielectric elastomer generator Appl. Phys. Lett. 94262902

[17] Koh S J A, Keplinger C, Li T, Bauer S and Suo Z 2011 Dielectric elastomer generators: how much energy can be converted? IEEE/ASME Trans. Mechatronics 16 33-41

[18] McKay T G 2011 Soft, low complexity dielectric elastomer generators Auckland Bioengineering Institute (Auckland: The University of Auckland)

[19] Brochu P, Yaun W, Zhang H and Pei Q 2009 Dielectric elastomers for direct wind-to-electricity power generation SMASIS2009 (Oxnard, CA: ASME) pp 1-7

[20] Jean-Mistral C, Basrour S and Chaillout J 2010 Modelling of dielectric polymers for energy scavenging applications Smart Mater. Struct. 19105006

[21] Hudak N S and Amatucci G G 2008 Small-scale energy harvesting through thermoelectric, vibration, and radiofrequency power conversion J. Appl. Phys. 103 101301

[22] Anderson I A, Rosset S, McKay T and Shea H 2014 Stack design for portable artificial muscle generators: is it dangerous to be short and fat? Proc. SPIE 90560Q 